\title{
Thalamus and focal to bilateral seizures
}

\section{A multiscale cognitive imaging study}

\author{
Lorenzo Caciagli, MD, PhD, Luke A. Allen, PhD, Xiaosong He, PhD, Karin Trimmel, MD, PhD, Sjoerd B. Vos, PhD, \\ Maria Centeno, MD, PhD, Marian Galovic, MD, Meneka K. Sidhu, MB, ChB, PhD, Pamela J. Thompson, PhD, \\ Danielle S. Bassett, PhD, Gavin P. Winston, BM BCh, PhD, FRCP, John S. Duncan, DM, FRCP, FMedSci, \\ Matthias J. Koepp, MD, PhD, FRCP, and Michael R. Sperling, MD
}

Neurology ${ }^{\circledR}$ 2020;95:e2427-e2441. doi:10.1212/WNL.0000000000010645

\begin{abstract}
\section{Objective}

To investigate the functional correlates of recurrent secondarily generalized seizures in temporal lobe epilepsy (TLE) using task-based fMRI as a framework to test for epilepsy-specific network rearrangements. Because the thalamus modulates propagation of temporal lobe onset seizures and promotes cortical synchronization during cognition, we hypothesized that occurrence of secondarily generalized seizures, i.e., focal to bilateral tonic-clonic seizures (FBTCS), would relate to thalamic dysfunction, altered connectivity, and whole-brain network centrality.
\end{abstract}

\section{Methods}

FBTCS occur in a third of patients with TLE and are a major determinant of disease severity. In this cross-sectional study, we analyzed 113 patients with drug-resistant TLE (55 left/58 right), who performed a verbal fluency fMRI task that elicited robust thalamic activation. Thirty-three patients $(29 \%)$ had experienced at least one FBTCS in the year preceding the investigation. We compared patients with TLE-FBTCS to those without FBTCS via a multiscale approach, entailing analysis of statistical parametric mapping (SPM) 12-derived measures of activation, task-modulated thalamic functional connectivity (psychophysiologic interaction), and graphtheoretical metrics of centrality.

\section{Results}

Individuals with TLE-FBTCS had less task-related activation of bilateral thalamus, with leftsided emphasis, and left hippocampus than those without FBTCS. In TLE-FBTCS, we also found greater task-related thalamotemporal and thalamomotor connectivity, and higher thalamic degree and betweenness centrality. Receiver operating characteristic curves, based on a combined thalamic functional marker, accurately discriminated individuals with and without FBTCS.

\section{Conclusions}

In TLE-FBTCS, impaired task-related thalamic recruitment coexists with enhanced thalamotemporal connectivity and whole-brain thalamic network embedding. Altered thalamic functional profiles are proposed as imaging biomarkers of active secondary generalization.

\author{
Correspondence \\ Dr. Caciagli \\ lorenzo.caciagli.11@ucl.ac.uk
}




\section{Glossary}

AED = antiepileptic drug; ANOVA = analysis of variance; AUC = area under the receiver operating characteristic curve; $\mathbf{C I}=$ confidence interval; FBTCS = focal to bilateral tonic-clonic seizures; FDR = false discovery rate; FS = focal seizures; FWE = familywise error; LTLE = left temporal lobe epilepsy; MANOVA = multivariate analysis of variance; NHNN = National Hospital for Neurology and Neurosurgery; PCA = principal component analysis; PPI = psychophysiologic interaction; ROC = receiver operating characteristic; ROI = region of interest; RTLE = right temporal lobe epilepsy; SMA = supplementary motor area; SUDEP = sudden unexpected death in epilepsy; svc = small volume correction; TLE = temporal lobe epilepsy.

Temporal lobe epilepsy (TLE) is the most common focal epilepsy syndrome in adults. Focal to bilateral tonic-clonic seizures (FBTCS), formerly termed secondarily generalized seizures, affect at least a third of people with TLE, ${ }^{1}$ are a major risk factor for seizure-related injuries and sudden unexpected death in epilepsy (SUDEP), ${ }^{2,3}$ and are a predictor of unfavorable postsurgical outcome. ${ }^{4}$ Why some people experience these seizures while others do not remains poorly understood. Presumably, specific functional and structural rearrangements may underlie the propensity for large-scale propagation of epileptic activity underlying this severe seizure type. Enhancing our understanding of the mechanisms leading to FBTCS may provide insight into much-needed novel therapeutic targets.

In TLE, thalamic atrophy represents the most common extratemporal abnormality ${ }^{5,6}$ and relates to derangements of cortico-subcortical connectivity, ${ }^{6,7}$ with unfavorable implications for postsurgical outcome. ${ }^{8,9}$ Converging evidence indicates that subcortical nuclei, particularly the thalamus, may be involved in the propagation of temporal lobe seizures. ${ }^{10,11}$ Resting-state fMRI analyses detected more widespread thalamocortical abnormalities in patients with TLE and FBTCS compared to those with focal seizures (FS) that do not generalize. ${ }^{12}$ Recent work also identified impairment of thalamotemporal structural connections in TLE-FBTCS. ${ }^{13}$

The thalamus contributes to motor planning, language, and memory by promoting cortical synchronization and facilitating cortico-cortical interplay. ${ }^{14,15}$ Cognitive tasks perturb brain network dynamics and evoke complex changes in interregional interactions, ${ }^{16}$ offering a powerful tool to identify disease-specific network traits ${ }^{17}$ that resting-state analyses may not adequately capture. ${ }^{18}$ In vivo, cognition is probed via task-based fMRI. Verbal fluency tasks assess expressive language and allow ascertaining language lateralization in focal epilepsy. ${ }^{19}$ Typical activation patterns encompass frontotemporo-parietal cortices, mesiotemporal structures, and, notably, bilateral thalamus, with left-sided emphasis. ${ }^{20,21}$

By challenging robustness of a functional network largely overlapping with the putative epileptogenic network of TLE, fluencyrelated task fMRI provides a powerful framework for assessing intergroup differences in underlying brain network organization. If the occurrence of FBTCS in TLE is related to abnormal thalamocortical interactions, one may expect to detect abnormal thalamic activation and connectivity with cognitive demand.
In this study we pursued a comprehensive characterization of the functional underpinnings of recurrent secondary generalization in TLE. As distinct from previous investigations, we envisioned the use of task-based fMRI to capture specific, FBTCS-associated rearrangements within networks recruited during linguistic processing. We hypothesized that, compared to TLE-FS, TLE with recent FBTCS would exhibit impaired thalamic activation, altered connectivity between thalamus and key symptomatogenic areas, including mesiotemporal and motor regions, and higher overall thalamic relevance for mediating signals within large-scale networks. To test these hypotheses, we employed a verbal fluency fMRI paradigm and a multiscale approach entailing comparison of TLE-FS and FBTCS across (1) task-related activation, (2) task-modulated changes of thalamic functional connectivity, via a psychophysiologic interaction analysis, and (3) graph-theoretical measures of thalamic centrality. We also linked domains of activation, connectivity, and centrality via a composite thalamic functional marker and investigated its potential to discriminate TLE-FS and FBTCS at the individual level.

\section{Methods}

\section{Participants}

For this cross-sectional investigation, we consecutively recruited 113 patients with drug-resistant TLE (55 left [30 female]; 58 right [43 female]), who underwent presurgical evaluation at the National Hospital for Neurology and Neurosurgery (NHNN), London, United Kingdom, between 2008 and 2013 (table 1). All participants underwent prolonged interictal and ictal scalp video-EEG, confirming and lateralizing the epileptic focus to one temporal lobe, and presurgical 3T MRI, with qualitative assessment and quantification of hippocampal volumetry and $\mathrm{T} 2$ relaxometry. ${ }^{22}$ Ipsilateral MRI findings included hippocampal sclerosis $(\mathrm{n}=32 / 29$, left TLE [LTLE]/right TLE [RTLE]), dysembryoplastic neuroepithelial tumor ( $n=5 / 8$, LTLE/RTLE), cavernoma ( $n=4 / 7$, LTLE/RTLE), and normal-appearing MRI ( $n=14 / 14$, LTLE/ RTLE). Contralateral mesiotemporal structures were normal in all cases. History of affective illness, referring to depressive and anxiety disorders, was recorded as detailed previously. ${ }^{23}$ Additional clinical/demographic details are available in appendix e-1 (doi.org/10.5061/dryad.2bvq83bm8).

Thirty-three patients (29.2\%; 20/13, LTLE/RTLE) had experienced at least 1 FBTCS during the year preceding the 
Table 1 Demographics, clinical characteristics, and neuropsychological test results

\begin{tabular}{|c|c|c|c|c|c|c|c|c|c|}
\hline & \multicolumn{3}{|c|}{ TLE $(n=113)$} & \multicolumn{3}{|c|}{ Left TLE $(n=55)$} & \multicolumn{3}{|c|}{ Right TLE $(n=58)$} \\
\hline & FS $(n=80)$ & $\begin{array}{l}\text { FBTCS } \\
(n=33)\end{array}$ & $\begin{array}{l}p \\
\text { Value }\end{array}$ & FS $(n=35)$ & $\begin{array}{l}\text { FBTCS } \\
(n=20)\end{array}$ & $\begin{array}{l}p \\
\text { Value }\end{array}$ & FS $(n=45)$ & $\begin{array}{l}\text { FBTCS } \\
(n=13)\end{array}$ & $\begin{array}{l}p \\
\text { Value }\end{array}$ \\
\hline Age, y, median (IQR) & $37.5(18.0)$ & $40.0(18.5)$ & 0.35 & $37.0(18.0)$ & $39.0(19.5)$ & 0.77 & $38.0(17.5)$ & $42.0(13.0)$ & 0.30 \\
\hline F/M & $54 / 26$ & 19/14 & 0.32 & 21/14 & 9/11 & 0.39 & $33 / 12$ & $10 / 3$ & 1.00 \\
\hline Handedness, L/R/ambidextrous & $10 / 66 / 4$ & $4 / 29 / 0$ & 0.42 & $4 / 30 / 1$ & $2 / 18 / 0$ & 1.00 & $6 / 36 / 3$ & $2 / 11 / 0$ & 1.00 \\
\hline Age at onset, $y$, median (IQR) & $17.0(15.0)$ & $14.0(13.5)$ & 0.73 & $18.0(16.0)$ & $15.0(13.5)$ & 0.75 & $17.0(15.5)$ & $14.0(16.5)$ & 0.90 \\
\hline $\begin{array}{l}\text { Epilepsy duration, y, median } \\
\text { (IQR) }\end{array}$ & $16.0(20.3)$ & $17.0(26.0)$ & 0.47 & $17.0(17.0)$ & $14.0(30.3)$ & 1.00 & $16.0(24.5)$ & $22.0(18.5)$ & 0.33 \\
\hline $\begin{array}{l}\text { Monthly focal seizure } \\
\text { frequency log, mean (SD) }\end{array}$ & $0.9(0.5)$ & $0.9(0.7)$ & 0.99 & $0.94(0.5)$ & $0.90(0.6)$ & 0.80 & $0.86(0.5)$ & $0.89(0.8)$ & 0.88 \\
\hline Lesional MRI, yes/no & $61 / 19$ & $27 / 6$ & 0.52 & $28 / 7$ & $15 / 5$ & 0.74 & $33 / 12$ & $12 / 1$ & 0.26 \\
\hline $\begin{array}{l}\text { Lesion type, none/HS/DNET/ } \\
\text { CAV }\end{array}$ & $21 / 43 / 10 / 6$ & $6 / 19 / 3 / 5$ & 0.53 & $9 / 21 / 3 / 2$ & $5 / 11 / 2 / 2$ & 0.96 & $12 / 22 / 7 / 4$ & $1 / 8 / 1 / 3$ & 0.26 \\
\hline AEDs, median (IQR) & $2.0(1.0)$ & $2.0(1.0)$ & 0.58 & $2.0(1.0)$ & $2.0(1.0)$ & 0.81 & $2.0(1.0)$ & $3.0(1.0)$ & 0.23 \\
\hline TPM/ZNS, yes/no & $16 / 64$ & $6 / 27$ & 1.00 & $10 / 25$ & $4 / 16$ & 0.54 & $6 / 39$ & $2 / 11$ & 1.00 \\
\hline LEV, yes/no & $42 / 38$ & $21 / 12$ & 0.31 & 18/17 & $14 / 6$ & 0.26 & $24 / 21$ & $7 / 6$ & 1.00 \\
\hline Benzodiazepines, yes/no & $26 / 54$ & $10 / 23$ & 1.00 & $11 / 24$ & $5 / 15$ & 0.76 & $15 / 30$ & $5 / 8$ & 0.75 \\
\hline $\begin{array}{l}\text { Hippocampal volume, } \\
\text { ipsilateral, mL, mean (SD) }\end{array}$ & $2.29(0.6)$ & $2.18(0.6)$ & 0.42 & $2.16(0.6)$ & $2.19(0.7)$ & 0.87 & $2.38(0.6)$ & $2.17(0.4)$ & 0.24 \\
\hline $\begin{array}{l}\text { Hippocampal volume, } \\
\text { contralateral, mL, mean (SD) }\end{array}$ & $2.78(0.3)$ & $2.76(0.4)$ & 0.83 & $2.77(0.3)$ & $2.83(0.4)$ & 0.52 & $2.79(0.2)$ & $2.67(0.3)$ & 0.17 \\
\hline $\begin{array}{l}\text { Thalamic volume, ipsilateral, } \\
\mathrm{mL} \text {, mean (SD) }\end{array}$ & $6.15(0.6)$ & $6.08(0.6)$ & 0.52 & $6.17(0.6)$ & $5.94(0.7)$ & 0.19 & $6.13(0.5)$ & $6.30(0.5)$ & 0.32 \\
\hline $\begin{array}{l}\text { Thalamic volume, contralateral, } \\
\mathrm{mL} \text {, mean (SD) }\end{array}$ & $6.29(0.5)$ & $6.21(0.5)$ & 0.40 & $6.32(0.5)$ & $6.19(0.6)$ & 0.36 & $6.27(0.4)$ & $6.25(0.3)$ & 0.86 \\
\hline NART IQ, mean (SD) & $99.2(11.9)$ & $101.3(15.0)$ & 0.38 & $97.3(12.2)$ & $101.7(14.6)$ & 0.25 & $100.8(11.7)$ & $100.5(16.5)$ & 0.95 \\
\hline Letter fluency, mean (SD) & $13.0(5.6)$ & $15.2(5.2)$ & 0.06 & $11.2(4.6)$ & $15.7(4.6)$ & $0.01^{a}$ & $14.3(6.0)$ & $14.4(6.2)$ & 0.95 \\
\hline Category fluency, mean (SD) & $18.1(5.1)$ & $18.9(5.9)$ & 0.55 & $17.7(4.5)$ & $19.8(5.8)$ & 0.14 & $18.5(5.5)$ & $17.3(5.9)$ & 0.50 \\
\hline Graded naming, mean (SD) & $15.5(5.6)$ & $14.5(6.3)$ & 0.73 & $13.7(5.7)$ & $13.4(6.5)$ & 0.84 & $17.0(5.1)$ & $16.4(5.6)$ & 0.75 \\
\hline Trail-Making Test A, mean (SD) & $35.3(13.9)$ & $32.3(13.5)$ & 0.18 & $34.2(12.1)$ & $31.3(13.2)$ & 0.24 & $36.1(15.3)$ & $34.3(14.8)$ & 0.57 \\
\hline Trail-Making Test B, mean (SD) & $75.4(32.6)$ & $79.4(32.7)$ & 0.53 & $75.6(30.5)$ & $81.0(36.8)$ & 0.66 & $75.2(34.6)$ & $75.9(23.3)$ & 0.73 \\
\hline BDI-FS score, median (IQR) & $3.0(4.0)$ & $2.0(5.0)$ & 0.20 & $4.0(4.0)$ & $2.0(4.0)$ & 0.18 & $3.0(3.3)$ & $2.0(6.5)$ & 0.62 \\
\hline BAI score, median (IQR) & $10.0(13.5)$ & $8.50(15.0)$ & 0.72 & $10.0(13.0)$ & $4.0(17.0)$ & 0.15 & $10.0(14.5)$ & $15.0(10.5)$ & 0.41 \\
\hline $\begin{array}{l}\text { Lifetime history of affective } \\
\text { disorder, yes/no }\end{array}$ & $29 / 51$ & $15 / 18$ & 0.40 & $14 / 21$ & $8 / 12$ & 1.00 & $15 / 30$ & $7 / 6$ & 0.21 \\
\hline LI (frontal ROI), median (IQR) & $0.75(0.4)$ & $0.78(0.4)$ & 0.23 & $0.65(0.3)$ & $0.78(0.4)$ & 0.09 & $0.78(0.5)$ & $0.63(0.5)$ & 0.89 \\
\hline LI (thalamus), median (IQR) & $0.52(0.6)$ & $0.49(0.4)$ & 0.96 & $0.55(0.4)$ & $0.48(0.4)$ & 0.62 & $0.45(0.7)$ & $0.56(0.5)$ & 0.71 \\
\hline $\begin{array}{l}\text { Framewise displacement, mm, } \\
\text { mean (SD) }\end{array}$ & $0.09(0.06)$ & $0.10(0.06)$ & 0.27 & $0.09(0.06)$ & $0.09(0.04)$ & 0.79 & $0.09(0.06)$ & $0.11(0.08)$ & 0.18 \\
\hline
\end{tabular}

Abbreviations: AED = antiepileptic drug; BAI = Beck Anxiety Inventory; BDI-FS = Beck Depression Inventory-Fast Screen; CAV = cavernoma; DNET = dysembryoplastic neuroepithelial tumor; FBTCS = focal to bilateral tonic-clonic seizures; FS = focal seizures only; HS = hippocampal sclerosis; IQR = interquartile range; $\mathrm{LI}=$ laterality index; NART = National Adult Reading Test; ROI = region of interest; TLE = temporal lobe epilepsy; TPM = topiramate; ZNS = zonisamide. Neuropsychological measures are reported as raw scores. Letter and category fluency data were missing for 4 patients. Statistics for Trail-Making Test A and B were carried out on log-transformed data, but raw data are provided in the table to ensure comparability with published literature. Framewise displacement values were computed according to the formula by Jenkinson and collaborators, ${ }^{\text {13 }}$ implemented in DPARSF for statistical parametric mapping (SPM) 12 (doi. org/10.5061/dryad.2bvq83bm8)

a This one $p$ value (for letter fluency, left TLE-FBTCS vs left TLE-FS) is FDR-adjusted across 6 cognitive measures (IQ, letter fluency, category fluency, naming, Trail-Making Test $A$ and $B$; uncorrected $p$ value $=0.002$ ). The other $p$ values are uncorrected for multiple comparisons. 
investigation (median frequency/month: 0.46, interquartile range: 0.83 ), and were therefore considered as having a current tendency for FBTCS (TLE-FBTCS). This 1-year cutoff for subgroup allocation was envisioned to probe the neural correlates of recent, active secondary generalization, and relies on multiple lines of evidence specifically linking generalized seizures in the last year to SUDEP risk, ${ }^{2,3}$ or recommending assessment of seizure freedom in the last year for clinical outcome classification. ${ }^{24} \mathrm{We}$ also conducted post hoc analyses on 3 groups after subdividing the main TLE-FS group into (1) TLE without lifetime history of FBTCS (never FBTCS, $\mathrm{n}=$ $38 ; 14 / 24$, left/right) and (2) TLE with history of remote FBTCS, but none for $>1$ year before scanning (remote FBTCS, $\mathrm{n}=42 ; 21 / 21$, left/right).

\section{Standard protocol approvals, registrations, and patient consents}

This study was approved by the NHNN and UCL Institute of Neurology Joint Research Ethics Committee. Written informed consent was obtained from all participants.

\section{Data acquisition and fMRI task specifics}

All participants underwent neuropsychological tests measuring intellectual level (IQ), letter and category fluency, and visual confrontation naming. We also evaluated group comparability for processing speed and executive function (table 1 and appendix e-1, doi.org/10.5061/dryad.2bvq83bm8). The Beck Depression Inventory-Fast Screen and Beck Anxiety Inventory measured mood and anxiety. Handedness was determined using the Edinburgh Handedness Inventory. T1-weighted and fMRI data were acquired on a 3T GE (Milwaukee, WI) SignaHDx MRI scanner using previously described protocols (appendix e-1, doi.org/10.5061/dryad.2bvq83bm8). ${ }^{25}$ Automated hippocampal and thalamic volumetric measures were available for all participants. All participants performed a verbal fluency paradigm lasting 5 minutes, consisting of 30-second task blocks requiring participants to covertly generate words beginning with a visually presented letter $(\mathrm{A} / \mathrm{D} / \mathrm{E} / \mathrm{S} / \mathrm{W} ; 1$ letter per block, 5 blocks in total), alternating with 30 -second blocks of crosshair fixation. ${ }^{26}$

\section{Analysis of clinical and neuropsychological data}

For all main analyses on 2 TLE groups (TLE-FBTCS vs TLEFS), we used the Fisher exact test, 2-sample $t$ test, and MannWhitney $U$ test for categorical, continuous parametric, and nonparametric variables, respectively. Correction for multiple comparisons was attained with the false discovery rate (FDR) procedure. Additional analyses comparing patients with FS and FBTCS were separately carried out for LTLE and RTLE subgroups. Details regarding post hoc analyses on 3 groups (TLE-FBTCS, remote FBTCS, and never FBTCS) are provided at the end of the Methods.

\section{Imaging data analysis: fMRI activation}

We analyzed fMRI data with statistical parametric mapping (SPM) 12 using previously detailed pipelines (appendix e-1, doi.org/10.5061/dryad.2bvq83bm8). ${ }^{25}$ Four participants were excluded owing to corrupted field of view $(n=1)$ or excessive motion $(>|3| \mathrm{mm}$ or $|3|$ degrees overall; $\mathrm{n}=3)$. For each participant, we computed voxel-wise parameter estimates and contrast images for task-related activation, including motion parameters as confounds. At the second level, 1-sample $t$ tests assessed fluency-related effects across all participants. Two-sample $t$ tests assessed differences between TLE-FBTCS and TLE-FS, with lateralization of the epileptic focus as covariate. Subgroup analyses separately compared LTLE-FS to LTLE-FBTCS and RTLE-FS to RTLE-FBTCS. Age and sex were used as covariates in all group comparisons. Sensitivity analyses entailed repeat group comparisons with letter fluency scores as nuisance regressors. Task effects were thresholded at $p<0.05$, corrected for multiple comparisons (familywise error rate $[\mathrm{FWE}]$ ) across the whole brain. In view of our a priori hypotheses, group differences were considered significant at $p<0.05$, FWE-corrected within a region of interest (ROI) consisting of a 12-mm-diameter sphere (small volume correction [FWE-svc]) centered at the location of the maxima for thalamus, hippocampus, and motor areas (precentral gyrus, supplementary motor area $[\mathrm{SMA}])^{27}$ For completeness, we report whole-brain effects at an exploratory threshold of $p<0.005$ uncorrected with a 20-voxel minimum cluster-size threshold $(p<0.005, \mathrm{k}=20){ }^{28,29}$ To convey higher spatial details for our thalamic findings, locations of activation and group difference maxima were related to thalamic subnuclei using the digital version of the Morel stereotactic atlas of the human thalamus. ${ }^{30}$ Hemispheric dominance for frontal and thalamic activation was determined via laterality indices of statistical parametric maps (appendix e-1, doi.org/10.5061/dryad.2bvq83bm8).

\section{Multiple regression models on thalamic activation}

We assessed determinants of task-related thalamic activation via multiple regression models, conducted with $R$-3.4.4. We extracted parameter estimates of thalamic activation from an independent ROI, represented by the ventral anterior nucleus (parvocellular part) of the Morel atlas, ${ }^{30}$ and used the following independent variables: occurrence of FBTCS in the last year, focal seizure frequency $(\log )$, sex, handedness, lateralization of the epileptic focus, number of antiepileptic drugs (AEDs), and affective history. For dimensionality reduction, measures of verbal fluency (letter/category fluency) and disease load (age at onset, disease duration) were entered into principal component analyses (PCAs; appendix e-1, doi. org/10.5061/dryad.2bvq83bm8). Both first principal components ("fluency" and "chronicity") were then implemented as additional regressors.

\section{Task-related functional connectivity: psychophysiologic interactions (PPIs)}

We probed thalamic connectivity with a PPI analysis, ${ }^{31}$ testing whether connection strength between a prespecified seed region and other brain areas was modulated by task execution. Individual fMRI time-series were obtained from the 
preprocessed images using a 12-mm diameter sphere centered on individual, participant-specific left and right anterior thalamic peak activation voxels (appendix e-1, doi.org/10.5061/ dryad.2bvq83bm8). ${ }^{32}$ The PPI general linear model included 3 regressors: (1) main effect of the seed region (i.e., the functional time series), (2) task regressor (i.e., psychological factor, represented by the vector of the word-generation block onset), and (3) interaction of the former 2, representing a taskmodulated change in connectivity, or PPI. ${ }^{31}$ Motion parameters were included as nuisance regressors. One-sample $t$ tests identified areas exhibiting task-related connectivity changes with the thalamic seeds. Two-sample $t$ tests compared TLEFBTCS and TLE-FS groups, as well as left and right TLE subgroups. Main PPI effects were thresholded at $p<0.05$, FWE-corrected across the whole brain. In view of our a priori hypotheses, group differences were considered significant at $p<$ 0.05 , FWE-corrected within a $12 \mathrm{~mm}$-diameter sphere (FWE$\mathrm{svc)}$ centered at the maxima in the hippocampus and motor areas. ${ }^{27}$ For completeness, whole-brain effects are reported at an exploratory statistical threshold of $p<0.005, \mathrm{k}=20 .^{28,29}$

\section{Graph-theoretical analysis}

Further image processing included regression of nuisance variables, bandpass filtering $(0.01-0.1 \mathrm{~Hz})$, and removal of the superimposed blocked task structure via condition-specific regressors, in line with benchmark evidence (appendix e-1, doi. org/10.5061/dryad.2bvq83bm8). Regional parcellation was attained via the Brainnetome atlas (246 ROI). ${ }^{33}$ After extracting ROI-averaged time series, we computed absolute Pearson correlation coefficients for every possible ROI pair, obtaining a $246 \times 246$ connectivity matrix for each participant. Weighted matrices were thresholded and binarized at network densities between $5 \%$ and $40 \%$ in increments of $1 \%$, yielding 36 binary undirected graphs per participant. Bilateral thalamic parcels (regions 231/232, corresponding to a left/right anterior thalamic division) were identified as nodes for network statistics. We investigated measures of centrality (hubness), in light of their relevance for clinical outcome prediction in TLE. ${ }^{9}$ For each node at each network density, we computed (1) degree centrality, describing the number of connections of a given node, and (2) betweenness centrality, describing the frequency with which a given node is located on the shortest path between other node pairs. Differences in thalamic centrality between TLE-FBTCS and TLE-FS, and for left and right TLE subgroups, were assessed via (1) comparisons of mean metric values, obtained after averaging across densities, ${ }^{34}$ and followed up with (2) subsequent contrasts for each network density value for each metric. We used nonparametric permutation tests entailing 10,000 permutations for each comparison, which generated permuted $t$ statistic distributions with associated $p$ values, ${ }^{9}$ followed by FDR adjustment for multiple testing ( $p_{\text {FDR }}$ $<0.05$; appendix e-1, doi.org/10.5061/dryad.2bvq83bm8).

\section{Receiver operating characteristic (ROC) curves with thalamic functional markers}

ROC curves assessed the accuracy with which age- and sexadjusted thalamic functional metrics could discriminate between TLE-FBTCS and TLE-FS. Initial models implemented markers of activation, extracted from the left ventral anterior thalamic parcel of the Morel atlas. To characterize the additional contribution of connectivity and graph metrics, ROC curve analyses were repeated using a composite functional construct, obtained after PCA on measures of activation, task-based connectivity, and centrality (appendix e-1, doi.org/10.5061/dryad.2bvq83bm8). Logistic regressions quantified the additive discriminative potential of activation and connectivity metrics. Models were compared via likelihood ratio tests.

\section{Post hoc analyses on 3 TLE groups}

Post hoc analyses examined TLE with (current) FBTCS, TLE remote FBTCS, and TLE never FBTCS regarding parameter estimates of thalamic and hippocampal activation, thalamotemporal and thalamomotor task-related connectivity, degree and betweenness centrality. Across all analyses, we specifically tested the hypothesis that altered thalamic network embedding would relate to a current propensity for secondary generalization and, consequently, that there would be no significant differences between individuals with remote FBTCS and never FBTCS. Subgroups were compared via multivariate and univariate analysis of variance (ANOVA), along with nonparametric permutation ANOVA for graphtheoretical metrics. Extraction of activation and connectivity metrics and statistical procedures are detailed in appendix e-1 (doi.org/10.5061/dryad.2bvq83bm8).

\section{Data availability}

Data supporting our findings are available from the corresponding author upon reasonable request.

\section{Results}

\section{Demographic and clinical characteristics}

There were no differences between TLE-FS and TLE-FBTCS for demographic and clinical variables, including temporal pathology subtype, number of AEDs, and usage of topiramate or zonisamide, which both affect verbal fluency activations ${ }^{28}$ (all $p>0.05$; table 1). Subgroup analyses, comparing LTLEFBTCS against LTLE-FS, and RTLE-FBTCS against RTLEFS, identified no significant differences. Propensity for FBTCS was similar in LTLE and RTLE subgroups $(\chi 2=2.66$, $p=0.10)$. A history of comorbid affective disorders was documented for $36.3 \%$ and $45.5 \%$ of patients with TLE-FS or TLE-FBTCS, respectively, with no group differences. Scores for anxiety and depression symptoms and usage of antidepressant/anxiolytic medication did not differ between groups (table 1 and appendix e-2, doi.org/10.5061/dryad. 2bvq 83 bm 8 ).

\section{Cognitive measures and volumetric findings}

There were no differences between patients with TLEFBTCS and patients with TLE-FS for all cognitive measures and thalamic and hippocampal volumes (all $p>0.05$; table 1 ). 
Subgroup analyses detected a difference between LTLEFBTCS and LTLE-FS regarding letter fluency scores, with LTLE-FBTCS outperforming LTLE-FS $\left(p_{\mathrm{FDR}}=0.01\right)$. Consequently, sensitivity analyses addressed confounding effects of fluency performance on imaging metrics. Additional analyses indicated that differences in letter fluency between LTLE-FS and LTLE-FBTCS were largely mediated by hippocampal volume, processing speed, and medication, all of which had no influence on thalamic activation, connectivity, and graph-theoretical metrics (linear regression models, all variables $p>0.23$; appendix e-2, doi.org/10.5061/dryad. $2 \mathrm{bvq} 83 \mathrm{bm} 8$ ). There were no other significant differences for cognitive and volumetric measures between LTLE and RTLE subgroups.

\section{Verbal fluency fMRI: activation-based analysis} The task elicited the expected ${ }^{28}$ activation of languagerelevant fronto-temporo-parietal cortices, hippocampus, putamen, and pallidum with left-sided emphasis, as well as right cerebellum (figure 1A). Thalamic activation encompassed bilateral anterior divisions and left-sided posterior nuclei, with local maxima in the ventral anterior parcel of the Morel atlas.

Patients with TLE-FBTCS had less task-related activation of bilateral anterior and posterior thalamus and left anterior hippocampus than patients with TLE-FS $(p<0.05$, FWE-svc; figure $1 \mathrm{~B}$ and table 2). Peak thalamic activation differences fell within ventral anterior nuclei; additional peaks were detected in the centrolateral/lateral posterior group. Exploratory wholebrain analyses detected lower activation in TLE-FBTCS in bilateral posterior parahippocampal gyrus and subcortical structures including putamen, pallidum, cerebellum, and subthalamus (figure 1B, second row). Sensitivity analyses controlling for fluency performance did not affect anterior thalamic findings, but reduced significance of hippocampal and right posterior thalamic differences (table 2). There was no increased activation in TLE-FBTCS compared to TLE-FS.

Post hoc analyses contrasted TLE subgroups with a sample of 53 healthy controls, balanced for demographic variables (appendix e-1, doi.org/10.5061/dryad.2bvq83bm8). Thalamic activation was comparable to controls in TLE-FS, and significantly lower in TLE-FBTCS (all $p_{\text {FDR }}<0.0003$ ), while hippocampal activation appeared reduced in both groups, with subtle effects in TLE-FS $\left(p=0.015\right.$, uncorrected; $\left.p_{\text {FDR }}=0.075\right)$, and marked changes in TLE-FBTCS $\left(p_{\mathrm{FDR}}<0.0001\right.$; figure e- 1 and appendix e-2, doi.org/10.5061/dryad.2bvq83bm8).

Subgroup analyses detected reduced activation of bilateral anterior thalamus, left posterior thalamus, and bilateral hippocampus in LTLE-FBTCS compared to LTLE-FS $(p<0.05$, FWE-svc; figure 1C and table e-1, doi.org/10.5061/dryad. $2 \mathrm{bvq} 83 \mathrm{bm} 8$ ). The subregional distribution of thalamic differences was similar to the main analysis, with ventral anterior maxima, and exploratory whole-brain comparisons in LTLEFBTCS showed hypoactivation of the same widespread subcortical areas described for the main analysis. Repeat models controlling for fluency performance did not affect subgroup findings. In RTLE, thalamic differences between FBTCS and FS were not significant (figure 1D).

Collectively, our findings indicate thalamic and hippocampal hypoactivation on verbal fluency fMRI in TLE-FBTCS.

\section{Multiple regression analysis on activation metrics}

Multiple regression based on the full predictor set was significant $\left(F_{9,93}=3.17, p=0.002\right.$; multiple $R^{2}=0.23$, adjusted $R^{2}=$ $0.16)$. Occurrence of FBTCS in the last year was the most significant determinant of thalamic activation, and the association was negative $(\beta=-0.17,95 \%$ confidence interval $[\mathrm{CI}]$ $[-0.28,-0.05], t=-2.90, p=0.005)$. Handedness, sex, and side of epilepsy also had significant effects $(\beta s=-0.19 /-0.13$ / $-0.11,95 \%$ CI $[-0.35,-0.04] /[-0.24,-0.01] /[-0.22,-0.005]$, $t=-2.53 /-2.24 /-2.06, p=0.013 / 0.027 / 0.041$, respectively). Interaction terms (FBTCS*handedness, FBTCS*lateralization, FBTCS $^{*}$ sex) were nonsignificant (all $p>0.05$ ).

\section{Psychophysiologic interaction analysis}

PPI analysis showed task-modulated connectivity changes between the left thalamic ROI and fronto-temporo-parietal cortices, contralateral thalamus, basal ganglia, and mesiotemporal lobes (figure 2A). Overlapping effects were identified for PPI analysis from the right thalamus (figure $3 \mathrm{~A}$ ). In both cases, task-modulated changes in connectivity were negative, implying reduced thalamic functional connectivity (i.e., thalamocortical decoupling) as a function of task performance, in accord with previous evidence. ${ }^{35}$

Compared to TLE-FS, TLE-FBTCS exhibited less attenuated task-dependent connectivity (i.e., failure to reduce coupling) between left thalamus and both left hippocampus and motor areas, including bilateral precentral gyrus and right SMA $(p<0.05$, FWE-svc; figure $2 \mathrm{~B}$ and table 2). Additional whole-brain effects were detected in left posterior insula/operculum, right superior frontal, and anterior cingulate cortices. Stronger task-dependent left thalamic connectivity to left hippocampus, contralateral thalamus, and motor areas was observed in LTLE-FBTCS compared to LTLE-FS, whereas significant differences only encompassed thalamo-motor connections in RTLE-FBTCS vs FS $(p<0.05$, FWE-svc; figure 2, C and D, and table e-1, doi.org/10.5061/dryad. 2bvq83bm8). Controlling for verbal fluency performance increased statistical significance of all group comparisons (table 2 and table e-1, doi.org/10.5061/dryad.2bvq83bm8).

Similarly, PPI analyses from the right thalamus highlighted less attenuated connectivity to left hippocampus and amygdala in TLE-FBTCS compared to TLE-FS ( $p<0.05$, FWE-svc; figure $3 \mathrm{~B}$ and table 2). Subgroup analyses showed higher connectivity to the left hippocampus in LTLE-FBTCS compared to LTLEFS, and additional whole-brain effects were identified for the right putamen (figure 3C and table e-1, doi.org/10.5061/ dryad.2bvq83bm8). In RTLE-FBTCS, stronger connectivity to 
A. Task effects
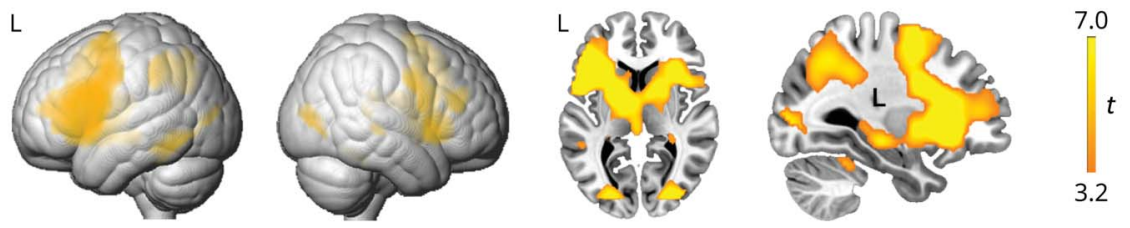

B. TLE - FBTCS < FS
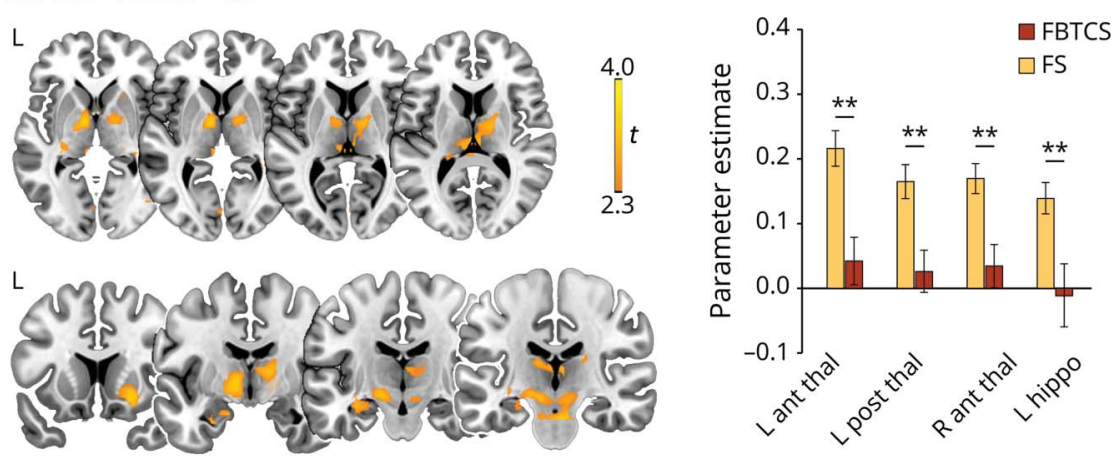

C. LTLE - FBTCS < FS
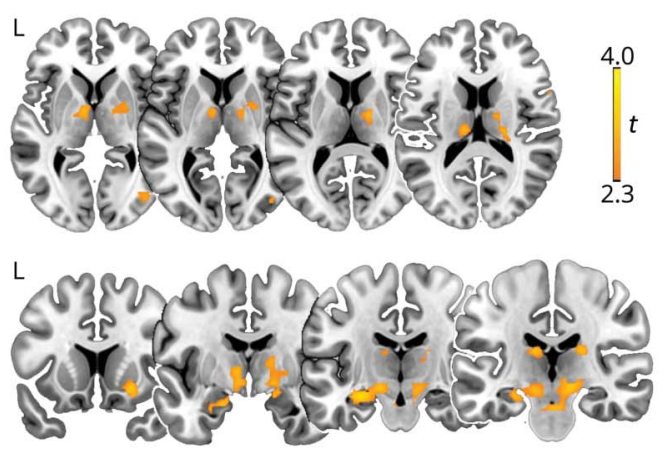

D. RTLE - FBTCS < FS

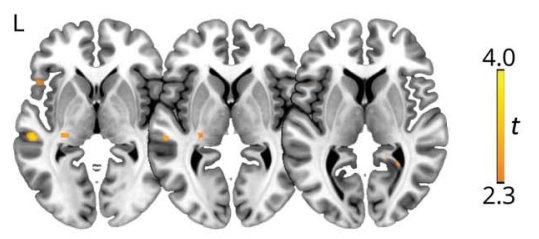

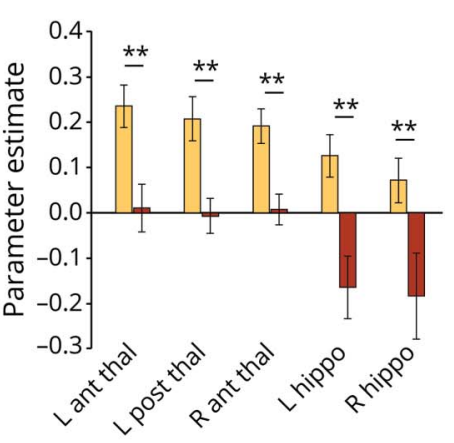

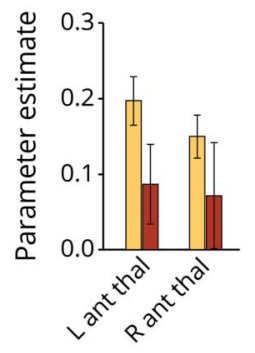

(A) Whole-brain verbal fluency activations across all participants, as derived from 1-sample $t$ tests. Axial and sagittal slices highlight activation of the thalamus, basal ganglia, and hippocampus. (B-D) Comparisons between temporal lobe epilepsy (TLE)-focal seizures (FS) and TLE-focal to bilateral tonic-clonic seizures (FBTCS) for task-related activation (B), and repeat contrasts for the same subgroups in left TLE (LTLE) (C) and right TLE (RTLE) (D). Axial slices specifically highlight differences in thalamic activation. Across $B$ to $D$, bar graphs display statistical parametric mappingderived parameter estimates of thalamic activation for areas of peak intergroup differences, namely left anterior/posterior thalamus, right anterior thalamus, and left hippocampus, for TLEFBTCS vs TLE-FS (B); all the former plus right hippocampus for LTLE subgroups (C); and left/right anterior thalamus for RTLE subgroups (D); in the latter case, thalamic activation differences did not reach statistical significance, but bar graphs are reported for completeness. Rendered images in A are thresholded at $p<0.05$, familywise error (FWE)-corrected for multiple comparisons across the whole brain. Across all panels, heat maps refer to brain slices, and display $t$ scores. Montreal Neurological Institute coordinates and $p$ values for group comparisons are provided in table 2 and table e-1 (doi.org/10.5061/dryad.2bvq83bm8). In bar graphs: $* * p<0.05$, FWE-small volume correction for peak intergroup difference. left amygdala and right hippocampus was evident at uncorrected thresholds. Sensitivity analyses controlling for linguistic performance amplified the above-described effects.

Collectively, our results point to enhanced task-related thalamotemporal and thalamo-motor interactions in TLEFBTCS.

\section{Graph-theoretical findings}

TLE-FBTCS showed significantly higher mean betweenness centrality of bilateral thalamus and higher mean right degree compared to TLE-FS (uncorrected $p=0.037 / 0.033 / 0.032$, respectively; all $p_{F D R}=0.049$, adjusted across 4 measures). Differences for left degree were not significant $\left(p_{F D R}=0.10\right)$. Regarding the former significant measures, higher centrality in TLE-FBTCS was apparent across most network densities (figure 4). Subgroup analyses showed higher left thalamic betweenness centrality in LTLE-FBTCS compared to FS at the uncorrected level, both for mean values ( $p=0.029$ uncorrected, $p_{\mathrm{FDR}}=0.12$ ) and across network densities, and significantly higher right thalamic degree in RTLE-FBTCS vs RTLE-FS $\left(p=0.008\right.$ uncorrected, $\left.p_{\mathrm{FDR}}=0.030\right)$. Inspection of plots for the remaining nonsignificant comparisons showed overall trends for higher centrality in FBTCS subgroups. 
Table 2 Comparisons of temporal lobe epilepsy (TLE)-focal to bilateral tonic-clonic seizures (FBTCS) and TLE-focal seizures (FS) for verbal fluency activation and psychophysiologic interaction (PPI) analyses: anatomic locations and statistical descriptors

\begin{tabular}{|c|c|c|c|c|c|c|}
\hline & \multicolumn{3}{|l|}{ Left hemisphere } & \multicolumn{3}{|l|}{ Right hemisphere } \\
\hline & $\begin{array}{l}\text { MNI coordinates } \\
(x, y, z)\end{array}$ & $Z$ score & $p$ Value & $\begin{array}{l}\text { MNI coordinates } \\
(x, y, z)\end{array}$ & $Z$ score & $p$ Value \\
\hline \multicolumn{7}{|l|}{$\begin{array}{l}\text { Verbal fluency activations, TLE-FS > } \\
\text { FBTCS }\end{array}$} \\
\hline Anterior thalamus (ventral anterior) & $-9,-4,-1$ & $\begin{array}{l}3.35 \\
(3.13)\end{array}$ & $0.005^{\mathrm{a}}\left(0.01^{\mathrm{a}}\right)$ & $15,-7,8$ & $\begin{array}{l}3.09 \\
(2.65)\end{array}$ & $0.011^{a}\left(0.034^{a}\right)$ \\
\hline $\begin{array}{l}\text { Posterior thalamus (centrolateral/ } \\
\text { lateral posterior) }\end{array}$ & $-12,-22,14$ & $\begin{array}{l}2.94 \\
(2.71)\end{array}$ & $0.016^{a}\left(0.029^{a}\right)$ & $6,-22,8$ & $\begin{array}{l}2.88 \\
(2.30)\end{array}$ & $0.019^{a}(0.071)$ \\
\hline Posterior thalamus (medial geniculate) & $-15,-28,-7$ & $\begin{array}{l}2.84 \\
(2.35)\end{array}$ & $0.021^{a}(0.065)$ & & & \\
\hline Hippocampus & $-30,-16,-16$ & $\begin{array}{l}2.82 \\
(2.30)\end{array}$ & $0.022^{\mathrm{a}}(0.078)$ & & & \\
\hline Putamen & & & & $24,8,-13$ & $\begin{array}{l}3.29 \\
(3.04)\end{array}$ & $0.001(0.001)$ \\
\hline Pallidum & $-15,-10,-7$ & $\begin{array}{l}3.36 \\
(2.72)\end{array}$ & $<0.001(0.003)$ & & & \\
\hline Ventral diencephalon (subthalamus) & $-12,-19,-10$ & $\begin{array}{l}3.14 \\
(2.67)\end{array}$ & $0.001(0.004)$ & $6,-28,-10$ & $\begin{array}{l}3.51 \\
(2.59)\end{array}$ & $<0.001(0.005)$ \\
\hline Posterior parahippocampal gyrus & $-15,-37,-4$ & $\begin{array}{l}3.28 \\
(2.80)\end{array}$ & $0.001(0.003)$ & $18,-34,-7$ & $\begin{array}{l}3.70 \\
(2.96)\end{array}$ & $<0.001(0.002)$ \\
\hline Cerebellum & & & & $6,55,-10$ & $\begin{array}{l}3.18 \\
(3.04)\end{array}$ & $0.001(0.001)$ \\
\hline \multicolumn{7}{|l|}{ Left thalamic PPI TLE-FBTCS > FS } \\
\hline Left hippocampus & $-36,-19,-16$ & $\begin{array}{l}2.77 \\
(3.13)\end{array}$ & $0.028^{\mathrm{a}}\left(0.011^{\mathrm{a}}\right)$ & & & \\
\hline Precentral gyrus & $-21,-16,53$ & $\begin{array}{l}2.53 \\
(3.31)\end{array}$ & $0.049^{\mathrm{a}}\left(0.006^{\mathrm{a}}\right)$ & $27,-19,65$ & $\begin{array}{l}2.78 \\
(3.13)\end{array}$ & $0.027^{a}\left(0.011^{a}\right)$ \\
\hline Supplementary motor area & & & & $3,11,50$ & $\begin{array}{l}2.70 \\
(3.31)\end{array}$ & $0.033^{\mathrm{a}}\left(0.006^{\mathrm{a}}\right)$ \\
\hline Superior frontal gyrus & & & & $18,20,41$ & $\begin{array}{l}3.22 \\
(3.61)\end{array}$ & $0.001(<0.001)$ \\
\hline Anterior cingulate cortex & & & & $3,32,8$ & $\begin{array}{l}2.92 \\
(3.01)\end{array}$ & $0.002(0.001)$ \\
\hline Insula/posterior parietal operculum & $-36,-25,29$ & $\begin{array}{l}3.37 \\
(3.72)\end{array}$ & $<0.001(<0.001)$ & & & \\
\hline \multicolumn{7}{|l|}{ Right thalamic PPI TLE-FBTCS > FS } \\
\hline Hippocampus & $-27,-25,16$ & $\begin{array}{l}2.91 \\
(3.09)\end{array}$ & $0.018^{\mathrm{a}}\left(0.011^{\mathrm{a}}\right)$ & & & \\
\hline Amygdala & $-27,-1,-22$ & $\begin{array}{l}2.91 \\
(3.11)\end{array}$ & $0.019^{\mathrm{a}}\left(0.010^{\mathrm{a}}\right)$ & & & \\
\hline
\end{tabular}

Abbreviation: Montreal Neurological Institute.

Coordinates of groupwise activation and PPI differences are given in MNI space. Coordinates of groupwise peak left thalamic activation for seed-based left PP analysis: $x=-9, y=-4, z=-8, z$ score $=$ infinite; coordinates of groupwise peak right thalamic activation for seed-based right PPI analysis: $x=9, y=-1, z=5$, $z$ score $=7.49$. $z$ Scores and $p$ values within parentheses are those obtained via sensitivity analyses using letter fluency scores as nuisance regressors. ${ }^{a}$ Statistically significant $p$ values $(p<0.05$, familywise error [FWE]-svc corrected) for peak-level differences.

For thalamic and hippocampal activation, thalamotemporal, and thalamo-motor connectivity, $p$ values were FWE-corrected for multiple comparisons using a 12-mm diameter spherical region of interest (svc) centered on the local maximum. Other $p$ values are uncorrected for multiple comparisons across the whole brain. 


\section{A. Task-modulated connectivity}
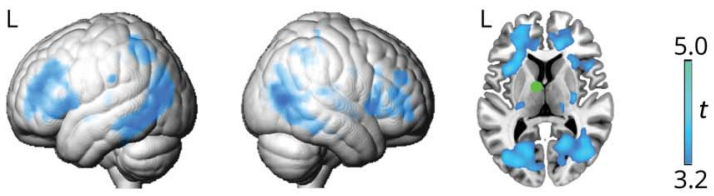

B. TLE - FBTCS > FS
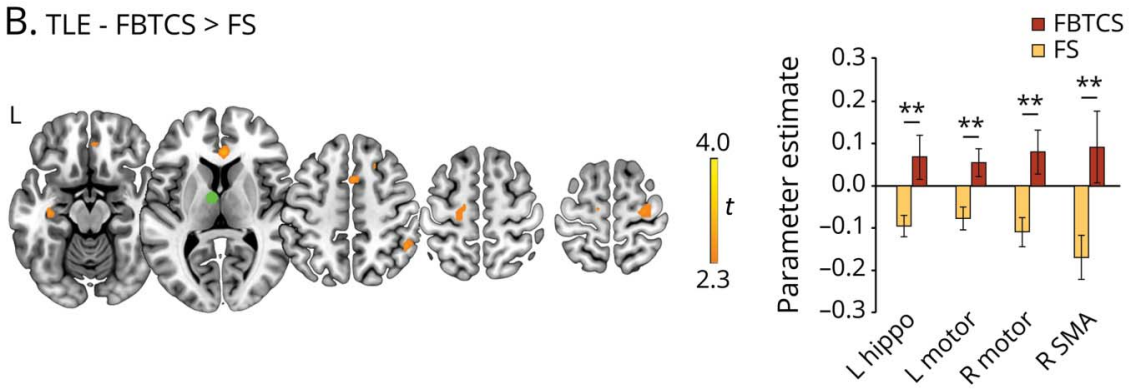

\section{LTLE - FBTCS > FS}

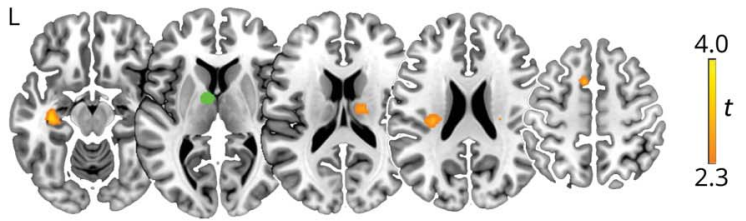

D. RTLE - FBTCS > FS

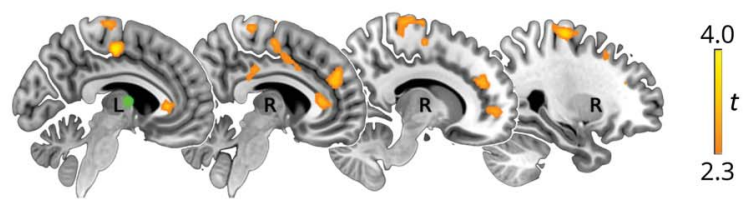

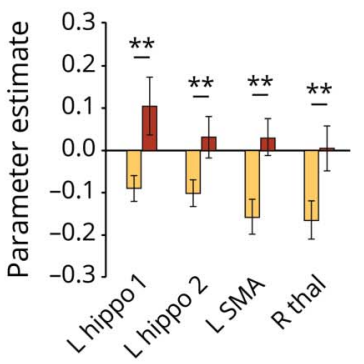

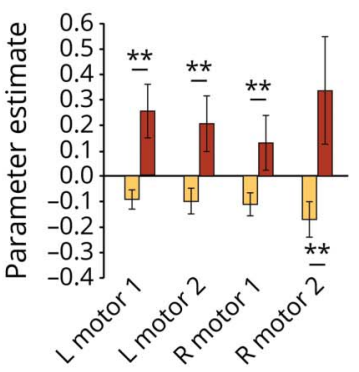

(A) Task-modulated changes in left anterior thalamic connectivity across all participants. The green sphere in the axial slice corresponds to the left thalamic seed. (B-D) Comparisons between temporal lobe epilepsy (TLE)-focal to bilateral tonic-clonic seizures (FBTCS) and TLE-focal seizures (FS) (B), and repeat contrasts for the same subgroups in left TLE (LTLE) (C) and right TLE (RTLE) (D). Across $B$ to $D$, bar graphs on the right display statistical parametric mapping (SPM)-derived parameter estimates of left thalamic PPI for areas of peak intergroup differences, namely left hippocampus, left/right precentral gyrus (motor cortex), and right supplementary motor area (SMA) for TLE-FBTCS vs TLE-FS (B); left hippocampus (2 spatially noncontiguous peaks), left SMA, and right medial dorsal thalamus for LTLE subgroups (C); left/right precentral gyrus (motor cortex; 2 spatially noncontiguous peaks on both sides) for RTLE subgroups (D). Rendered images in $A$ are thresholded at $p<0.001$, uncorrected for illustration purposes. Across all panels, heat maps refer to brain slices, and display $t$ scores. Montreal Neurological Institute coordinates and $p$ values for group comparisons are provided in table 2 and table e-1 (doi.org/ 10.5061/dryad.2bvq83bm8). In bar graphs: $\star \star p<0.05$, familywise error-small volume correction for peak between-group difference.

\section{Individual discrimination via thalamic functional measures}

ROC curve analyses based on left anterior thalamic activation discriminated between TLE-FBTCS and TLE-FS (area under the ROC curve [AUC] 0.67 [95\% CI 0.56-0.77], $p=0.007$ ). Subgroup analyses detected higher discrimination of LTLE subgroups (AUC 0.69 [0.55-0.83], $p=0.026$ ), while findings in RTLE approached significance (AUC 0.67 [0.52-0.83], $p=0.06)$. Use of a composite functional marker, incorporating activation, task-related connectivity, and graphtheory metrics, achieved substantially higher discrimination than activation measures alone (ROC curve on combined metric, AUC 0.75 [0.64-0.85], $p<0.0001)$. Effects were more prominent for LTLE (AUC 0.83 [0.70-0.95], $p=0.0001$ ), and also significant in RTLE (AUC 0.73 [0.58-0.89], $p=$ 0.011). Comparison of logistic regressions via likelihood ratio tests (appendix e-1, doi.org/10.5061/dryad.2bvq83bm8) identified marked additive contributions of task-related connectivity to subgroup discrimination $(p=0.006)$, whereas addition of graph-theoretical metrics to the former 2 only yielded marginal improvements in model fit $(p>0.10)$.

\section{Post hoc analyses on 3 TLE groups}

Multivariate ANOVA (MANOVA) on measures of activation, left, and right PPI identified no significant differences between TLE never FBTCS and remote FBTCS ( $p=0.25$ / $0.60 / 0.63$, respectively; $p>0.23$ for all univariate analyses). MANOVA on 3 groups, on the other hand, confirmed significant effects for thalamic activity and, left and right PPI $\left(p=0.016 / 0.021 / 0.013\right.$, respectively; all $p_{\mathrm{FDR}}=$ 0.021 ), with corrected univariate post hoc analyses (Tukey range test) detecting differences for comparison of 


\section{A. Task-modulated connectivity}
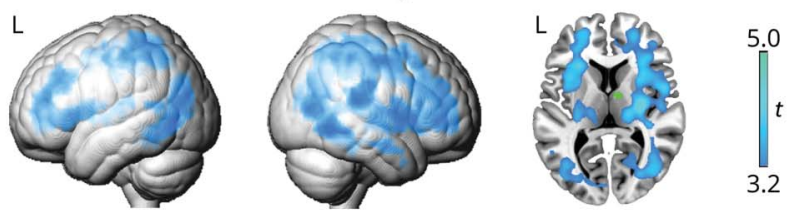

B. TLE - FBTCS > FS
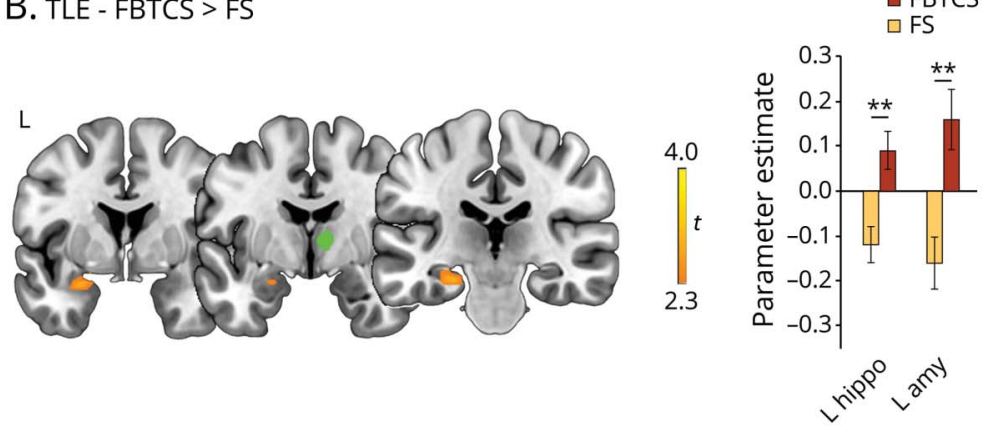

C. LTLE - FBTCS > FS

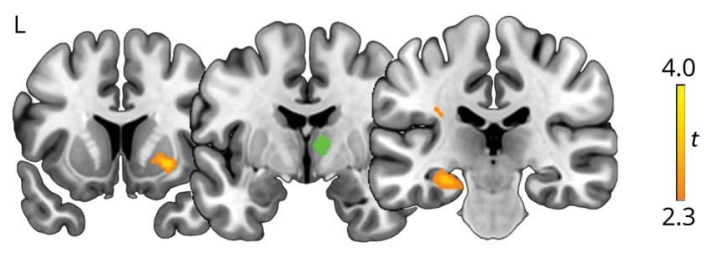

D. RTLE - FBTCS > FS

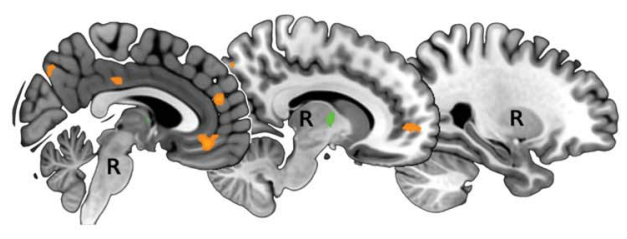

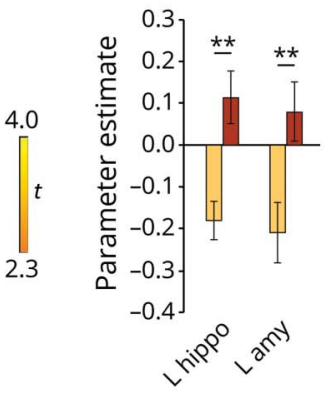

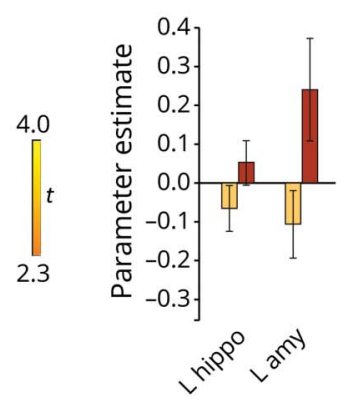

(A) Task-modulated changes in right anterior thalamic connectivity across all participants. The green sphere in the axial slice shows the upper portion of the right thalamic seed. (B-D) Comparisons between temporal lobe epilepsy (TLE)-focal to bilateral tonic-clonic seizures (FBTCS) and TLE-focal seizures (FS) (B), and repeat contrasts for the same subgroups in left TLE (LTLE) (C) and right TLE (RTLE) (D). For B to D, bar graphs on the right display statistical parametric mapping (SPM)-derived parameter estimates of right thalamic PPI for areas of peak intergroup differences, corresponding to left hippocampus and left amygdala for all group comparisons. As for analyses in RTLE, bar graphs are reported for completeness, but group differences for hippocampal and amygdala activity did not reach statistical significance. Rendered images in A are thresholded at $p<0.001$ uncorrected for illustration purposes. Across all panels, heat maps refer to brain slices, and display $t$ scores. Montreal Neurological Institute coordinates and $p$ values for group comparisons are provided in table 2 and table e-1 (doi.org/10.5061/dryad. 2bvq83bm8). In bar graphs: ** $p<0.05$, familywise error-small volume correction for peak between-group difference.
TLE-FBTCS vs either TLE never FBTCS or TLE remote FBTCS or both (figure 5 and table e-2, doi.org/10.5061/ dryad.2bvq 83 bm 8$)$.

Analysis of thalamic graph-theoretical metrics via nonparametric ANOVA highlighted uncorrected group effects for bilateral betweenness centrality and right degree (figure 5). Post hoc tests indicated no statistically significant differences between TLE never FBTCS and TLE remote FBTCS for any metric at any network density level (all $p>0.05$, uncorrected across network densities within each metric). Plot inspection confirmed the previously documented pattern of higher centrality in TLE-FBTCS. Separate analyses for LTLE/RTLE subgroups are described in figure e-2 and appendix e-2 (doi. org/10.5061/dryad.2bvq83bm8).

\section{Discussion}

In TLE, previous research documented thalamic involvement during temporal lobe seizures ${ }^{11,36}$ and identified thalamic atrophy 5,37 along with altered structural and functional connectivity. ${ }^{38-40}$ While much research focused on TLE as a whole, few investigations sought to identify markers of propensity for secondary generalization, and no studies investigated thalamic activation and connectivity during cognitive tasks. Using a verbal fluency fMRI paradigm, we document coexistence of attenuated thalamic and hippocampal activation with stronger task-modulated thalamotemporal connectivity and higher thalamic centrality in TLE with active FBTCS, compared to TLE with focal seizures only. Current presence of FBTCS was defined based on the occurrence of such seizures in the year preceding the 


\section{A. TLE - FBTCS > FS}

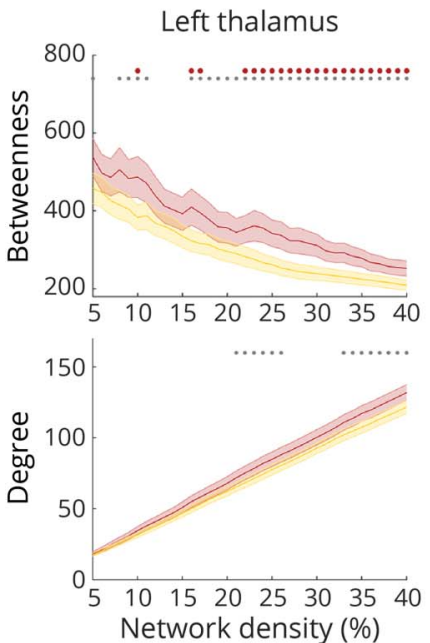

C. RTLE - FBTCS > FS
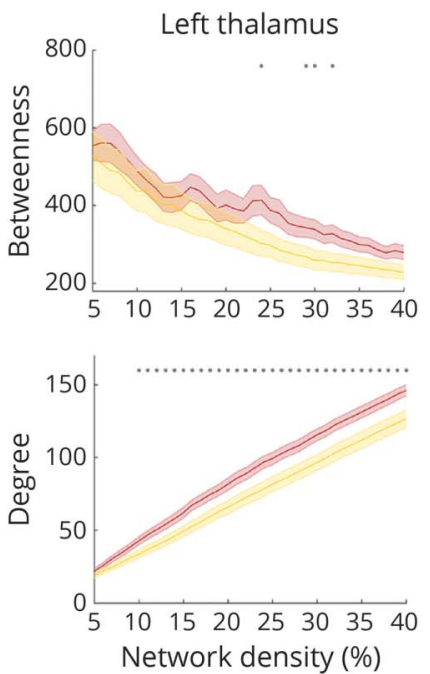

B. LTLE - FBTCS > FS

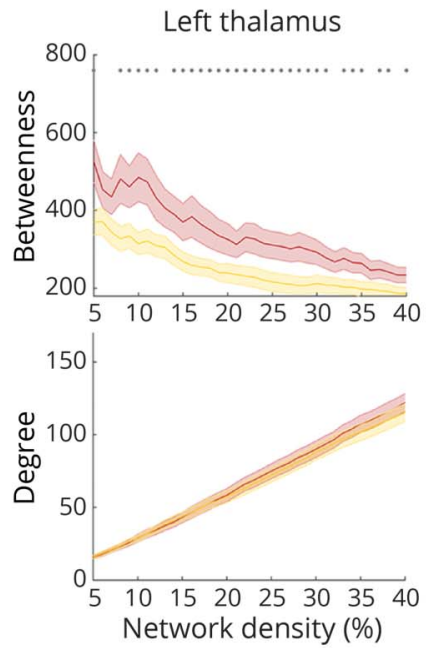

(A-C) Measures of betweenness and degree centrality of the left and right thalamic region of interest. Metrics for focal to bilateral tonic-clonic seizures (FBTCS) and focal seizures (FS) patient groups are displayed with dark red and orange lines, respectively. Shaded bands display standard errors, red dots indicate significant intergroup differences after false discovery rate correction for multiple comparisons $\left(p_{\text {FDR }}<0.05\right)$, gray dots indicate between-group differences at $p<0.05$, uncorrected for multiple comparisons. Statistical details for comparisons of mean graph-theoretical metrics are provided in the main text. LTLE $=$ left temporal lobe epilepsy; RTLE = right temporal lobe epilepsy; TLE = temporal lobe epilepsy.

investigation, in accordance with established clinical recommendations. ${ }^{2,3,24}$ Post hoc comparisons of patients with a history of remote FBTCS vs those with no lifetime experience of secondary generalization detected no significant differences in thalamic profiles, suggesting that the identified thalamic functional abnormalities specifically relate to the presence of active, uncontrolled FBTCS. By challenging a functional network largely overlapping with the putative epileptogenic network of TLE, our findings indicate impaired thalamic functional profiles as potential candidate markers of recurrent FBTCS, and thus disease severity.

Analysis of task-related activation detected reduced anterior and posterior thalamic recruitment in TLE-FBTCS compared to TLE-FS, with greater significance on the left. Hippocampal activation was also lower in TLE-FBTCS. Corroborating our a priori hypotheses, these findings indicate task-related disengagement of key components of the pathologic network of TLE in the subgroup with FBTCS, emphasizing the involvement of the thalamus, and advancing preliminary evidence of suboptimal hippocampal recruitment during language in TLE. ${ }^{26}$ From a neurobiological perspective, the fMRI signal relates to local field potentials, and likely reflects the extent of incoming input and local processes. ${ }^{41}$ Hence, we hypothesize that repeated insults of secondarily generalized epileptic activity may lead to more marked derangements of local neural activity and affect richness of synaptic connections, which may in turn explain impaired task-related recruitment of both hippocampus and thalamus in TLE-FBTCS. Discrepancies of effects emerging from the 


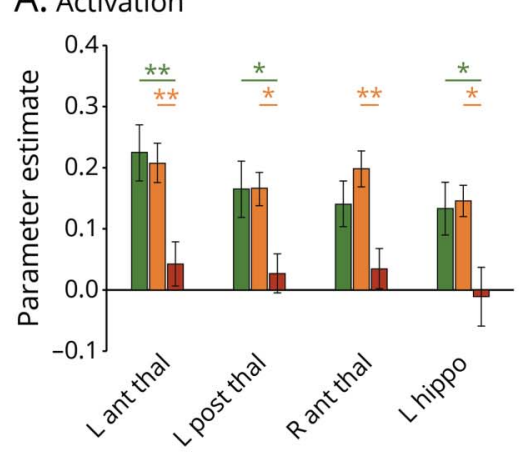

B. Left PPI

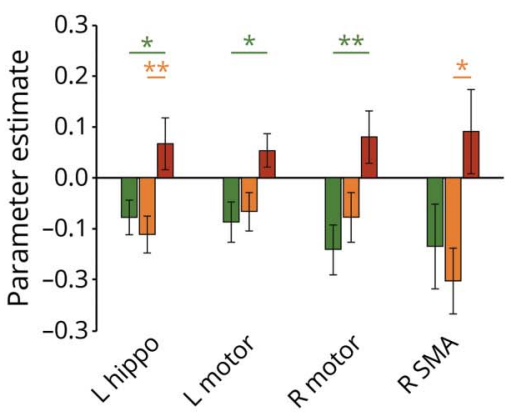

C. Right PPI

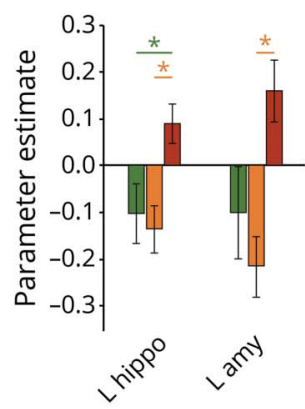

D. Centrality

Left thalamus
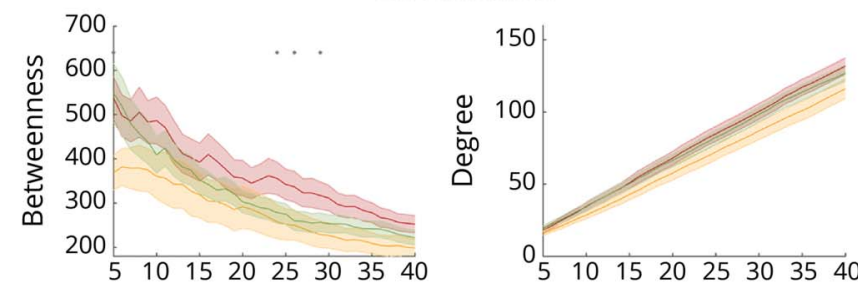

Right thalamus
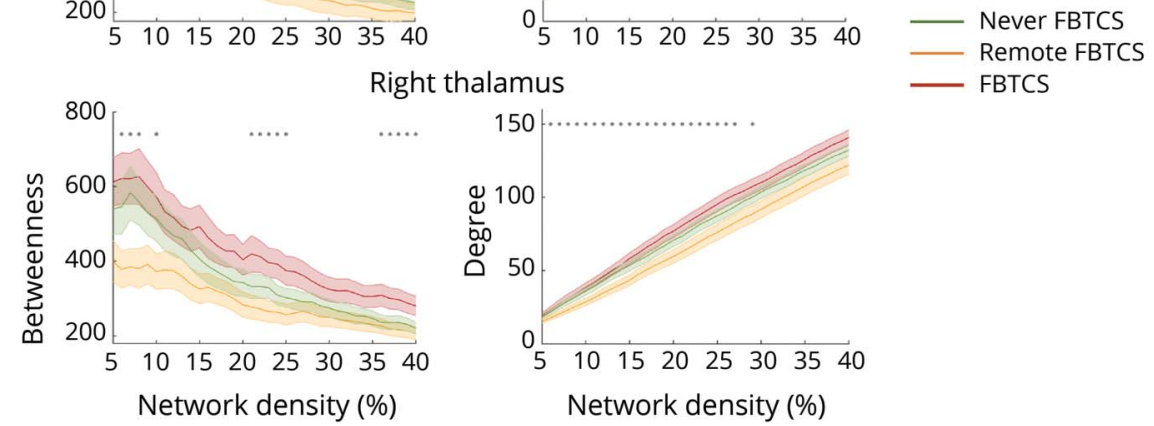

(A-D) Comparisons among (1) TLE with (current) focal to bilateral tonic-clonic seizures (FBTCS), corresponding to the group termed TLE-FBTCS throughout the article, (2) TLE remote FBTCS and (3) TLE never FBTCS (for further grouping details, see Methods). Bar graphs in A to C display parameter estimates extracted from locations of peak group differences in the main analysis on 2 groups, corresponding to: left anterior/posterior thalamus, right anterior thalamus, and left hippocampus for thalamic activation (A); left hippocampus, left/right precentral gyrus (motor cortex) and right supplementary motor area (SMA) for left thalamic psychophysiologic interaction (PPI) (B); left hippocampus and amygdala for right thalamic PPI (C). Montreal Neurological Institute coordinates of each location are provided in table 2 and e-1 (doi.org/10.5061/dryad.2bvq83bm8). (D) Group comparisons for measures of betweenness and degree centrality of the left and right thalamic region of interest. Shaded bands display standard errors, gray dots indicate between-group differences at $p<0.05$, uncorrected for multiple comparisons. There were no significant intergroup differences after correction for multiple testing. In bar graphs: ${ }^{*} p<0.01$, corrected (Tukey); * $p<0.05$, corrected (Tukey).

comparisons between left and right TLE subgroups may relate to task specifics, as verbal fluency fMRI paradigms implicate linguistic processing, and are particularly suited to capture effects within left hemispheric networks. ${ }^{42}$ Sensitivity analyses, including fluency scores as nuisance regressor, did not affect the results of the main group comparison and subgroup analyses, indicating that hippocampal and thalamic disengagement may occur during cognitive effort, but be independent of cognitive performance levels. We further confirmed subgroup comparability across a large series of clinical and demographic factors, including frontal and thalamic laterality indices. Moreover, multiple regression models identified FBTCS as the most significant determinant of anterior thalamic activation, among an extensive set of demographic, clinical, and cognitive measures.
Analysis of fMRI activation identifies areas implicated in task execution, but does not formally capture the interplay between those areas, known as functional connectivity. To assess thalamotemporal connectivity during task-based fMRI, we conducted a PPI analysis, providing measures of contextdependent, task-modulated changes in coupling between a seed region and the whole brain. ${ }^{31}$ PPI analysis from both left and right thalamus demonstrated attenuation of task-related connectivity to fronto-temporo-parietal cortices and subcortical targets, in accordance with previous results in healthy controls. ${ }^{35}$ Supporting a modulatory role of the thalamus during executive cognition, these findings relate to neurophysiologic studies indicating thalamus-driven synchronization and mediation of cortico-cortical information transfer. ${ }^{43}$ 
Group comparisons highlighted abnormal thalamotemporal interactions in TLE-FBTCS compared to FS, with less attenuated task-related connectivity between thalami and left hippocampus in the FBTCS subgroup, and altered connections between left thalamus and right anterior cingulate cortex. Stronger thalamotemporal coherence was particularly evident for comparisons of left TLE subgroups, while RTLEFBTCS exhibited higher connectivity between thalamus and motor areas compared to RTLE-FS. Previous resting-state fMRI work in TLE documented bilaterally impaired connectivity of the posterior thalamus in TLE-FBTCS, ${ }^{12}$ but correlated thalamic time courses with those of cortical parcels with near-lobar extent. Here, we found that FBTCS relate to state-dependent connectivity differences affecting key components of the pathologic network of TLE, including limbic and rolandic areas. Task-based connectivity analysis thus provides an important complement to activation-based comparisons, by showing that reduced activation of hippocampus and thalamus is underpinned by stronger interregional synchrony and failure of reciprocal disengagement during cognition. From a mechanistic viewpoint, these findings may imply a reduced adaptability of neural communications within circuitry underlying secondary generalization, and highlight an association between recurrent FBTCS and more stereotyped, inflexible patterns of network interactions.

Graph-theoretical analysis allows tracking the organizational properties of brain networks, and centrality measures identify network hubs, i.e., regions with high connectivity to other network nodes and prominent influence over global network dynamics. In TLE, graph-theory investigations identified abnormalities of both mesiotemporal ${ }^{44}$ and whole-brain network architecture. ${ }^{17}$ Aberrant nodal topology was documented for limbic regions as well as thalamus, ${ }^{45}$ and recent work reported higher thalamic centrality as predictor of postsurgical seizure recurrence. ${ }^{9}$ Here, we identified higher anterior thalamic centrality in TLE-FBTCS compared to TLE-FS during a verbal fluency task, further supporting a relationship between FBTCS and higher thalamic functional integration within whole-brain networks. Our graph-theoretical results provide a third line of evidence for altered thalamic network embedding in TLEFBTCS relative to TLE-FS. Higher centrality likely implies stronger connectional profiles and enhanced thalamic relevance within the context of whole-brain network architecture, ${ }^{9}$ which may underpin a network configuration facilitating diffuse dissemination of ictal discharges, and thus recurrent FBTCS.

To assess the potential clinical relevance of thalamic functional markers, we employed those within ROC curve analyses probing discrimination of TLE-FS and TLE-FBTCS. Although models already conveyed significant results with activation measures alone, discrimination abilities were substantially enhanced after combining measures of activity, connectivity, and centrality into a composite thalamic functional construct, reaching $75 \%$ accuracy for all TLE and $>80 \%$ in LTLE. While proving the advantage of combining imaging metrics derived across investigative scales, these findings directly implicate thalamic functional profiles as potential surrogate marker of secondary generalization, with validity at the individual level.

Overall, our results dovetail with evidence from animal models, documenting the pivotal role of impaired thalamic gating for propagation and maintenance of seizures involving the neocortex, ${ }^{46}$ and the efficacy of thalamotomy in suppressing the latter. ${ }^{47}$ In patients with TLE, high-frequency thalamic stimulation desynchronizes hippocampal and large-scale epileptic network activity and induces cortico-cortical decoupling, ${ }^{48}$ which may underlie the efficacy of deep brain anterior thalamic stimulation. ${ }^{49}$ Our findings also complement recent restingstate $\mathrm{AMRI}$ evidence for abnormal interactions between thalamic divisions and basal ganglia in TLE with recent FBTCS. ${ }^{50}$ While differing methodologically, both analyses compellingly indicate a prominent role of the thalamus in shaping susceptibility to uncontrolled secondary generalization in TLE.

Our task-based fMRI investigation indicates reduced thalamic activation coupled with enhanced thalamotemporal connectivity and whole-brain thalamic network embedding as a functional signature of recurrent FBTCS in TLE. These patterns appear dynamic, and specifically relate to the presence of recent, uncontrolled secondary generalization. Altered thalamic network engagement is proposed as an imaging biomarker of active FBTCS, and thus disease severity, in TLE. While shedding light on the potential network correlates of recurrent FBTCS, our study delivers a viable target to track individual response to treatment and assess efficacy of novel therapeutic strategies directed toward generalization of focal seizures and SUDEP.

\section{Acknowledgment}

The authors thank the patients for their participation; Philippa Bartlett, Jane Burdett, and Elaine Williams, radiographers at the Epilepsy Society MRI Unit, for help during data acquisition; Jason Stretton for help with data acquisition; Drs. Britta Wandschneider and Urs Braun for comments on an earlier version of the manuscript; Dr. Peter Zeidman (UCL Functional Imaging Group) for methodologic input regarding psychophysiologic interaction analysis; and Dr. Aidan O’Keeffe for statistical advice.

\section{Study funding}

The study was funded through the Wellcome Trust (project grants 079474 and 083148). The Epilepsy Society supported the Epilepsy Society MRI scanner. This research was also supported by the National Institute for Health Research University College London Hospitals Biomedical Research Centre. L. Caciagli was funded by a $\mathrm{PhD}$ scholarship from Brain Research UK (award reference 14181) and acknowledges previous support by an Ermenegildo Zegna Founder's Scholarship. K. Trimmel was supported by fellowships of the European Academy of Neurology and the Austrian Society of Neurology (OEGN). M.K. Sidhu is supported by the National Institute for Health Research University College London Hospitals Biomedical Research Centre. D.S. Bassett acknowledges support from the John D. and Catherine T. 
MacArthur Foundation, the Alfred P. Sloan Foundation, the ISI Foundation, and NINDS R01-NS099348-01. G.P. Winston was supported by an MRC Clinician Scientist Fellowship $(\mathrm{MR} / \mathrm{M} 00841 \mathrm{X} / 1)$. The funders had no role in study design, data collection and analysis, decision to publish, or preparation of the manuscript.

\section{Disclosure}

L. Caciagli, L.A. Allen, X. He, K. Trimmel, S.B. Vos, M. Centeno, M. Galovic, M.K. Sidhu, P.J. Thompson, and D.S. Bassett report no disclosures relevant to the manuscript. G.P. Winston reports grant funding from the Medical Research Council during the conduct of the study. J.S. Duncan and M.J. Koepp report no disclosures relevant to the manuscript. M.R. Sperling reports grants from Medtronic, UCB Pharma, Takeda, Neurelis, SK Life Science, Engage Therapeutics, and Eisai outside the submitted work. Go to Neurology.org/ $\mathrm{N}$ for full disclosures.

\section{Publication history}

Received by Neurology December 2, 2019. Accepted in final form June 1, 2020.

\section{Appendix Authors}

\begin{tabular}{lll}
\hline Name & Location & Contribution \\
\hline $\begin{array}{ll}\text { Lorenzo } \\
\text { Caciagli, MD, }\end{array}$ & $\begin{array}{l}\text { Queen Square, London, } \\
\text { and Chalfont St Peter, UK }\end{array}$ & $\begin{array}{l}\text { Study concept and design, } \\
\text { analysis and interpretation } \\
\text { of data including statistical } \\
\text { analysis, drafting and } \\
\text { revision of manuscript for } \\
\text { intellectual content }\end{array}$ \\
& &
\end{tabular}

\begin{tabular}{lll}
\hline $\begin{array}{l}\text { Luke A. Allen, } \\
\text { PhD }\end{array}$ & $\begin{array}{l}\text { Queen Square, London, } \\
\text { and Chalfont St Peter, UK }\end{array}$ & $\begin{array}{l}\text { Analysis of data, revision of } \\
\text { manuscript for intellectual } \\
\text { content }\end{array}$
\end{tabular}

\begin{tabular}{ll}
\hline $\begin{array}{l}\text { Xiaosong He, } \\
\text { PhD }\end{array}$ & $\begin{array}{l}\text { University of } \\
\text { Pennsylvania, } \\
\text { Philadelphia }\end{array}$ \\
\hline $\begin{array}{l}\text { Karin } \\
\text { Trimmel, MD, } \\
\text { PhD }\end{array}$ & $\begin{array}{l}\text { Queen Square, London, } \\
\text { and Chalfont St Peter, UK, } \\
\text { and Vienna, Austria }\end{array}$ \\
\hline $\begin{array}{l}\text { Sjoerd B. Vos, } \\
\text { PhD }\end{array}$ & $\begin{array}{l}\text { Queen Square, London, } \\
\text { and Chalfont St Peter, UK }\end{array}$ \\
\end{tabular}

Interpretation of data, revision of manuscript for intellectual content

Interpretation of data, revision of manuscript for intellectual content

Contribution of data analysis scripts, revision of manuscript for intellectual content

\begin{tabular}{lll}
\hline $\begin{array}{l}\text { Maria } \\
\text { Centeno, MD, } \\
\text { PhD }\end{array}$ & $\begin{array}{l}\text { Queen Square, London, } \\
\text { and Chalfont St Peter, UK }\end{array}$ & $\begin{array}{l}\text { Acquisition of data, } \\
\text { revision of manuscript for } \\
\text { intellectual content }\end{array}$ \\
\hline $\begin{array}{l}\text { Marian } \\
\text { Galovic, MD }\end{array}$ & $\begin{array}{l}\text { Queen Square, London, } \\
\text { and Chalfont St Peter, UK }\end{array}$ & $\begin{array}{l}\text { Revision of manuscript for } \\
\text { intellectual content }\end{array}$ \\
\hline $\begin{array}{l}\text { Meneka K. } \\
\text { Sidhu, } \\
\text { MBChB, PhD, } \\
\text { MRCP }\end{array}$ & $\begin{array}{l}\text { Queen Square, London, } \\
\text { and Chalfont St Peter, UK }\end{array}$ & $\begin{array}{l}\text { Acquisition of data, } \\
\text { revision of manuscript for } \\
\text { intellectual content }\end{array}$ \\
\hline $\begin{array}{l}\text { Pamela J. } \\
\text { Thompson, } \\
\text { PhD }\end{array}$ & $\begin{array}{l}\text { Queen Square, London, } \\
\text { and Chalfont St Peter, UK }\end{array}$ & $\begin{array}{l}\text { Acquisition of data, } \\
\text { revision of manuscript for } \\
\text { intellectual content }\end{array}$ \\
\hline $\begin{array}{l}\text { Danielle S. } \\
\text { Bassett, PhD }\end{array}$ & $\begin{array}{l}\text { University of } \\
\text { Pennsylvania, }\end{array}$ & $\begin{array}{l}\text { Consulting on statistics, } \\
\text { interpretation of data, } \\
\text { revision of manuscript for } \\
\text { intellectual content }\end{array}$ \\
& &
\end{tabular}

\section{Appendix (continued)}

\begin{tabular}{lll}
\hline Name & Location & Contribution \\
\hline $\begin{array}{l}\text { Gavin P. } \\
\text { Winston, BM } \\
\text { BCh, PhD, } \\
\text { MRCP }\end{array}$ & $\begin{array}{l}\text { Queen Square, London, } \\
\text { and Chalfont St Peter, UK, } \\
\text { and Kingston, Canada }\end{array}$ & $\begin{array}{l}\text { Acquisition of data, } \\
\text { revision of manuscript for } \\
\text { intellectual content }\end{array}$ \\
\hline $\begin{array}{l}\text { John S. } \\
\text { Duncan, DM, } \\
\text { FRCP }\end{array}$ & $\begin{array}{l}\text { Queen Square, London, } \\
\text { and Chalfont St Peter, UK }\end{array}$ & $\begin{array}{l}\text { Study supervision, } \\
\text { interpretation of data, } \\
\text { revision of manuscript for } \\
\text { intellectual content, } \\
\text { obtainment of funding }\end{array}$ \\
\hline $\begin{array}{l}\text { Matthias J. } \\
\text { Koepp, MD, } \\
\text { PhD, FRCP }\end{array}$ & $\begin{array}{l}\text { Queen Square, London, } \\
\text { and Chalfont St Peter, UK }\end{array}$ & $\begin{array}{l}\text { Study concept or design, } \\
\text { study supervision, } \\
\text { interpretation of data, } \\
\text { revision of manuscript for } \\
\text { intellectual content, } \\
\text { obtainment of funding }\end{array}$ \\
\hline $\begin{array}{l}\text { Michael R. } \\
\text { Sperling, MD }\end{array}$ & $\begin{array}{l}\text { Thomas Jefferson } \\
\text { University, Philadelphia, }\end{array}$ & $\begin{array}{l}\text { Study concept or design, } \\
\text { study supervision, analysis } \\
\text { and interpretation of data, } \\
\text { revision of manuscript for } \\
\text { intellectual content }\end{array}$ \\
\hline & &
\end{tabular}

\section{References}

1. Jobst BC, Williamson PD, Neuschwander TB, Darcey TM, Thadani VM, Roberts DW. Secondarily generalized seizures in mesial temporal epilepsy: clinical characteristics, lateralizing signs, and association with sleep-wake cycle. Epilepsia 2001;42: 1279-1287.

2. Walczak TS, Leppik IE, D'Amelio M, et al. Incidence and risk factors in sudden unexpected death in epilepsy: a prospective cohort study. Neurology 2001;56:519-525.

3. Nilsson L, Farahmand BY, Persson PG, Thiblin I, Tomson T. Risk factors for sudden unexpected death in epilepsy: a case-control study. Lancet 1999;353:888-893.

4. Janszky J, Janszky I, Schulz R, et al. Temporal lobe epilepsy with hippocampal sclerosis: predictors for long-term surgical outcome. Brain 2005;128:395-404.

5. Barron DS, Fox PM, Laird AR, Robinson JL, Fox PT. Thalamic medial dorsal nucleus atrophy in medial temporal lobe epilepsy: a VBM meta-analysis. NeuroImage Clin 2012;2:25-32.

6. Bernhardt BC, Bernasconi N, Kim H, Bernasconi A. Mapping thalamocortical network pathology in temporal lobe epilepsy. Neurology 2012;78:129-136.

7. Mueller SG, Laxer KD, Barakos J, et al. Involvement of the thalamocortical network in TLE with and without mesiotemporal sclerosis. Epilepsia 2010;51:1436-1445.

8. Keller SS, Richardson MP, Schoene-Bake JC, et al. Thalamotemporal alteration and postoperative seizures in temporal lobe epilepsy. Ann Neurol 2015;77:760-774.

9. He X, Doucet GE, Pustina D, Sperling MR, Sharan AD, Tracy JI. Presurgical thalamic "hubness" predicts surgical outcome in temporal lobe epilepsy. Neurology 2017;88: 2285-2293.

10. Norden $\mathrm{AD}$, Blumenfeld $\mathrm{H}$. The role of subcortical structures in human epilepsy. Epilepsy Behav 2002;3:219-231.

11. Bertram EH, Mangan PS, Zhang D, Scott CA, Williamson JM. The midline thalamus: alterations and a potential role in limbic epilepsy. Epilepsia 2001;42:967-978.

12. He X, Doucet GE, Sperling M, Sharan A, Tracy JI. Reduced thalamocortical functional connectivity in temporal lobe epilepsy. Epilepsia 2015;56:1571-1579.

13. Chen C, Li H, Ding F, et al. Alterations in the hippocampal-thalamic pathway underlying secondarily generalized tonic-clonic seizures in mesial temporal lobe epilepsy: a diffusion tensor imaging study. Epilepsia 2019;60:121-130.

14. Saalmann YB, Kastner S. The cognitive thalamus. Front Syst Neurosci 2015;9:39.

15. Schmitt LI, Wimmer RD, Nakajima M, Happ M, Mofakham S, Halassa MM. Thalamic amplification of cortical connectivity sustains attentional control. Nature 2017;545: 219-223.

16. Cole MW, Bassett DS, Power JD, Braver TS, Petersen SE. Intrinsic and task-evoked network architectures of the human brain. Neuron 2014;83:238-251.

17. Caciagli L, Bernhardt BC, Hong SJ, Bernasconi A, Bernasconi N. Functional network alterations and their structural substrate in drug-resistant epilepsy. Front Neurosci 2014;8:411.

18. He X, Bassett DS, Chaitanya G, Sperling MR, Kozlowski L, Tracy JI. Disrupted dynamic network reconfiguration of the language system in temporal lobe epilepsy. Brain 2018;141:1375-1389.

19. Szaflarski JP, Gloss D, Binder JR, et al. Practice guideline summary: use of fMRI in the presurgical evaluation of patients with epilepsy: report of the guideline development, dissemination, and implementation subcommittee of the American Academy of Neurology. Neurology 2017;88:395-402.

20. Wagner S, Sebastian A, Lieb K, Tuscher O, Tadic A. A coordinate-based ALE functional MRI meta-analysis of brain activation during verbal fluency tasks in healthy control subjects. BMC Neurosci 2014;15:19. 
21. Yarkoni T, Poldrack RA, Nichols TE, Van Essen DC, Wager TD. Large-scale automated synthesis of human functional neuroimaging data. Nat Methods 2011;8:665-670.

22. Winston GP, Cardoso MJ, Williams EJ, et al. Automated hippocampal segmentation in patients with epilepsy: available free online. Epilepsia 2013;54:2166-2173.

23. Stretton J, Pope RA, Winston GP, et al. Temporal lobe epilepsy and affective disorders: the role of the subgenual anterior cingulate cortex. J Neurol Neurosurg Psychiatry 2015;86:144-151.

24. Engel J Jr, Wiebe S, French J, et al. Practice parameter: temporal lobe and localized neocortical resections for epilepsy: report of the quality standards subcommittee of the American Academy of Neurology, in association with the American Epilepsy Society and the American Association of Neurological Surgeons. Neurology 2003;60: $538-547$.

25. Xiao F, Caciagli L, Wandschneider B, et al. Effects of carbamazepine and lamotrigine on functional magnetic resonance imaging cognitive networks. Epilepsia 2018;59: 1362-1371.

26. Bonelli SB, Powell R, Thompson PJ, et al. Hippocampal activation correlates with visual confrontation naming: fMRI findings in controls and patients with temporal lobe epilepsy. Epilepsy Res 2011;95:246-254.

27. Sidhu MK, Stretton J, Winston GP, et al. A functional magnetic resonance imaging study mapping the episodic memory encoding network in temporal lobe epilepsy. Brain 2013;136:1868-1888.

28. Wandschneider B, Burdett J, Townsend L, et al. Effect of topiramate and zonisamide on fMRI cognitive networks. Neurology 2017;88:1165-1171.

29. Lieberman MD, Cunningham WA. Type I and type II error concerns in fMRI research: re-balancing the scale. Soc Cogn Affect Neurosci 2009;4:423-428.

30. Krauth A, Blanc R, Poveda A, Jeanmonod D, Morel A, Szekely G. A mean threedimensional atlas of the human thalamus: generation from multiple histological data. Neuroimage 2010;49:2053-2062.

31. Friston KJ, Buechel C, Fink GR, Morris J, Rolls E, Dolan RJ. Psychophysiological and modulatory interactions in neuroimaging. NeuroImage 1997;6:218-229.

32. Zeidman P, Jafarian A, Corbin N, et al. A guide to group effective connectivity analysis, part 1: first level analysis with DCM for fMRI. Neuroimage 2019;200:174-190.

33. Fan $\mathrm{L}, \mathrm{Li} \mathrm{H}$, Zhuo J, et al. The human brainnetome atlas: a new brain atlas based on connectional architecture. Cereb Cortex 2016;26:3508-3526.

34. Bassett DS, Bullmore E, Verchinski BA, Mattay VS, Weinberger DR, Meyer-Lindenberg A. Hierarchical organization of human cortical networks in health and schizophrenia. J Neurosci 2008;28:9239-9248.
35. O'Muircheartaigh J, Vollmar C, Barker GJ, et al. Abnormal thalamocortical structural and functional connectivity in juvenile myoclonic epilepsy. Brain 2012;135: 3635-3644

36. Guye M, Regis J, Tamura M, et al. The role of corticothalamic coupling in human temporal lobe epilepsy. Brain 2006;129:1917-1928.

37. Keller SS, Roberts N. Voxel-based morphometry of temporal lobe epilepsy: an introduction and review of the literature. Epilepsia 2008;49:741-757.

38. Keller SS, O'Muircheartaigh J, Traynor C, Towgood K, Barker GJ, Richardson MP. Thalamotemporal impairment in temporal lobe epilepsy: a combined MRI analysis of structure, integrity, and connectivity. Epilepsia 2014;55:306-315.

39. Dinkelacker V, Valabregue R, Thivard L, et al. Hippocampal-thalamic wiring in medial temporal lobe epilepsy: enhanced connectivity per hippocampal voxel. Epilepsia 2015;56:1217-1226

40. Barron DS, Fox PT, Pardoe $\mathrm{H}$, et al. Thalamic functional connectivity predicts seizure laterality in individual TLE patients: application of a biomarker development strategy. NeuroImage Clin 2015;7:273-280.

41. Logothetis NK, Pauls J, Augath M, Trinath T, Oeltermann A. Neurophysiological investigation of the basis of the fMRI signal. Nature 2001;412:150-157.

42. Bonelli SB, Thompson PJ, Yogarajah M, et al. Imaging language networks before and after anterior temporal lobe resection: results of a longitudinal fMRI study. Epilepsia 2012;53:639-650.

43. Sherman SM. Thalamus plays a central role in ongoing cortical functioning. Nat Neurosci 2016;19:533-541.

44. Bernhardt BC, Bernasconi N, Hong SJ, Dery S, Bernasconi A. Subregional mesiotemporal network topology is altered in temporal lobe epilepsy. Cereb Cortex 2016; 26:3237-3248.

45. Vaughan DN, Rayner G, Tailby C, Jackson GD. MRI-negative temporal lobe epilepsy: a network disorder of neocortical connectivity. Neurology 2016;87:1934-1942.

46. Paz JT, Davidson TJ, Frechette ES, et al. Closed-loop optogenetic control of thalamus as a tool for interrupting seizures after cortical injury. Nat Neurosci 2013;16:64-70.

47. Mondragon S, Lamarche M. Suppression of motor seizures after specific thalamotomy in chronic epileptic monkeys. Epilepsy Res 1990;5:137-145.

48. Yu T, Wang X, Li Y, et al. High-frequency stimulation of anterior nucleus of thalamus desynchronizes epileptic network in humans. Brain 2018;141:2631-2643.

49. Salanova V, Witt $T$, Worth $R$, et al. Long-term efficacy and safety of thalamic stimulation for drug-resistant partial epilepsy. Neurology 2015;84:1017-1025.

50. He X, Chaitanya G, Asma B, et al. Disrupted basal ganglia-thalamocortical loops in focal to bilateral tonic-clonic seizures. Brain 2020;143:175-190. 


\section{Neurology}

\section{Thalamus and focal to bilateral seizures: A multiscale cognitive imaging study Lorenzo Caciagli, Luke A. Allen, Xiaosong He, et al. \\ Neurology 2020;95;e2427-e2441 Published Online before print August 26, 2020 \\ DOI 10.1212/WNL.0000000000010645}

This information is current as of August 26, 2020

Updated Information \& Services

References

Subspecialty Collections

Permissions \& Licensing

Reprints including high resolution figures, can be found at: http://n.neurology.org/content/95/17/e2427.full

This article cites 50 articles, 10 of which you can access for free at: http://n.neurology.org/content/95/17/e2427.full\#ref-list-1

This article, along with others on similar topics, appears in the following collection(s):

fMRI

http://n.neurology.org/cgi/collection/fmri

Information about reproducing this article in parts (figures,tables) or in its entirety can be found online at:

http://www.neurology.org/about/about_the_journal\#permissions

Information about ordering reprints can be found online:

http://n.neurology.org/subscribers/advertise

Neurology ${ }^{\circledR}$ is the official journal of the American Academy of Neurology. Published continuously since 1951, it is now a weekly with 48 issues per year. Copyright Copyright ( 2020 The Author(s). Published by Wolters Kluwer Health, Inc. on behalf of the American Academy of Neurology.. All rights reserved. Print ISSN: 0028-3878. Online ISSN: 1526-632X.

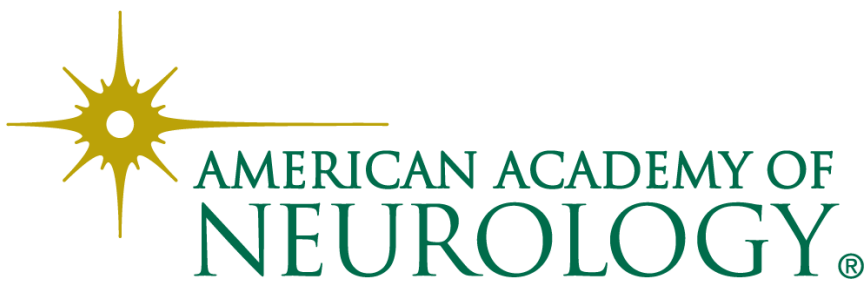

